\title{
PIk1 Phosphorylates CLIP-170 and Regulates Its Binding to Microtubules for Chromosome Alignment
}

\author{
Mai Kakeno $^{1}$, Kenji Matsuzawa ${ }^{1}$, Toshinori Matsui ${ }^{1}$, Hiroki Akita ${ }^{1}$, Ikuko Sugiyama ${ }^{1}$, \\ Fumiyoshi Ishidate ${ }^{1}$, Atsushi Nakano ${ }^{2}$, Seiji Takashima ${ }^{3}$, Hidemasa Goto ${ }^{4}$, Masaki Inagaki ${ }^{4}$, \\ Kozo Kaibuchi $^{1}$, and Takashi Watanabe ${ }^{1^{*}}$ \\ ${ }^{1}$ Department of Cell Pharmacology, Nagoya University Graduate School of Medicine, 65 Tsurumai, Showa, \\ Nagoya, Aichi 464-8550, Japan, ${ }^{2}$ Department of Clinical Medicine and Development, National Cerebral \\ and Cardiovascular Center, 5-7-1 Fujishirodai, Suita, Osaka 565-8565, Japan, ${ }^{3}$ Department of Medical \\ Biochemistry, Osaka University Graduate School of Medicine, 2-2 Yamadaoka, Suita, Osaka 565-0871, \\ Japan, ${ }^{4}$ Division of Biochemistry, Aichi Cancer Center Research Institute, 1-1 Kanokoden, Chikusa, \\ Nagoya, Aichi 464-8681, Japan
}

\begin{abstract}
The microtubule (MT) cytoskeleton is essential for cellular morphogenesis, cell migration, and cell division. MT organization is primarily mediated by a variety of MT-associated proteins. Among these proteins, plus-end-tracking proteins (+TIPs) are evolutionarily conserved factors that selectively accumulate at growing MT plus ends. Cytoplasmic linker protein (CLIP)-170 is a +TIP that associates with diverse proteins to determine the behavior of MT ends and their linkage to intracellular structures, including mitotic chromosomes. However, how CLIP-170 activity is spatially and temporally controlled is largely unknown. Here, we show that phosphorylation at Ser312 in the third serine-rich region of CLIP-170 is increased during mitosis. Polo-like kinase 1 (Plk1) is responsible for this phosphorylation during the mitotic phase of dividing cells. In vitro analysis using a purified CLIP-170 N-terminal fragment showed that phosphorylation by Plk1 diminishes CLIP-170 binding to the MT ends and lattice without affecting binding to EB3. Furthermore, we demonstrate that during mitosis, stable kinetochore/MT attachment and subsequent chromosome alignment require CLIP-170 and a proper phosphorylation/dephosphorylation cycle at Ser312. We propose that CLIP-170 phosphorylation by PIk1 regulates proper chromosome alignment by modulating the interaction between CLIP-170 and MTs in mitotic cells and that CLIP-170 activity is stringently controlled by its phosphorylation state, which depends on the cellular context.
\end{abstract}

Key words: +TIPs, CLIP-170, phosphorylation, Plk1, microtubules

\section{Introduction}

The microtubule (MT) cytoskeleton plays critical roles in a range of physiological phenomena, including directional cell

\footnotetext{
*To whom correspondence should be addressed: Takashi Watanabe, Department of Cell Pharmacology, Nagoya University Graduate School of Medicine, 65 Tsurumai, Showa, Nagoya, Aichi 464-8550, Japan.

Tel: +81-52-744-2075, Fax: +81-52-744-2083

E-mail: takashiw@med.nagoya-u.ac.jp

Abbreviations: AMPK, AMP-activated protein kinase; CAP-Gly, cytoskeleton-associated protein glycine-rich; Cdk1, cyclin-dependent kinase 1; CLIP-170, cytoplasmic linker protein-170; DTT, dithiothreitol; EBs, End binding proteins; FL, full length; GFP, green fluorescent protein; kMT, kinetochore microtubule; MAPs, microtubule-associated proteins; MT, microtubule; Plk1, polo-like kinase 1; STLC, S-trityl-L-cysteine; +TIP, plus-end-tracking protein; WT, wild type.
}

migration, proliferation, and morphogenesis. MTs are hollow tubes that are composed of 13 protofilaments with $\alpha$ - and $\beta$ tubulin heterodimers that align in a head-to-tail manner. Due to their intrinsic polarity, MTs contain two distinct ends: a slow-growing minus end and a fast-growing plus end (Howard and Hyman, 2003). In most cells, the minus ends of MTs are relatively static, whereas the plus ends are highly dynamic and oscillate between phases of growing and shortening. MT plus ends reach and form linkages with cellular structures, such as kinetochores in mitotic cells and the specialized cortex in migrating cells (Watanabe et al., 2005; Galjart, 2010; Gouveia and Akhmanova, 2010). MT architecture in cells is utilized as a template for organelle positioning, a scaffold for vesicular and protein trafficking, and a platform for intracellular signaling. 
A variety of microtubule-associated proteins (MAPs) determine MT organization in cells depending on intra- and extra-cellular signals. Among these MAPs, much attention has been directed to plus-end-tracking proteins (+TIPs), which preferentially accumulate at the growing plus ends of MTs. Intensive research has identified a number of +TIPs and revealed their functions in MT dynamics and MT-mediated physiological processes, including mitosis and vesicular transport (Galjart, 2010; Gouveia and Akhmanova, 2010). Furthermore, the development of in vitro methods to reconstitute +TIP behavior and high resolution structural studies have facilitated our understanding of how +TIPs localize at the growing ends of MTs (Akhmanova and Steinmetz, 2008; Slep, 2010). End binding proteins (EBs) autonomously accumulate at the growing ends of MTs by recognizing the nucleotide-dependent structural cap of the MT ends through their N-terminal calponin homology domain (Maurer et al., 2011, 2012). The C-terminal region of EBs recruits other +TIPs, including CLIP-170, CLASPs, and SLAIN2. These +TIPs harbor one of the two distinct EB-binding domains, the cytoskeletonassociated protein glycine-rich (CAP-Gly) domain or the SxIP motif (Honnappa et al., 2009), although several +TIPs localize to the plus ends independent of EBs. At the plus ends of growing MTs, each +TIP exerts its specific roles in MT dynamics and the linkages of MTs with intracellular structures in cooperation with other +TIPs (Akhmanova and Steinmetz, 2008; Galjart, 2010). Biochemical and biophysical characterization of each +TIP has cultivated our understanding of this process, but the molecular mechanisms governing the interactive networks among +TIPs and MTs has yet to be clarified.

CLIP-170 was identified as an ATP-sensitive MTbinding protein in cultured cells (Rickard and Kreis, 1990, 1991; Pierre et al., 1992). Studies have demonstrated that CLIP-170 connects MT ends to the following intracellular structures: actin filaments through IQGAP1 for cell migration (Fukata et al., 2002), chromosomes during cell division (Dujardin et al., 1998; Tanenbaum et al., 2006; Li et al., 2010), and organelle membranes for their intracellular transport (Rickard and Kreis, 1996; Lomakin et al., 2009). In addition, CLIP-170 was proposed to be a rescue factor that facilitates the phase conversion of MTs from shrinking to growing (Komarova et al., 2002), although, to date, this effect has failed to be reconstituted in vitro using purified proteins (Bieling et al., 2008; Dixit et al., 2009). The tandem CAP-Gly domains in the N-terminus of CLIP-170 primarily mediate its end-tracking by recognizing the acidic tails of EBs and tubulins (Honnappa et al., 2006; Mishima et al., 2007) and facilitate its intramolecular association with its own C-terminus, resulting in autoinhibition (Lansbergen et al., 2004). The C-terminal zinc-finger motifs of CLIP-170 mediate its localization at kinetochores for chromosome alignment in prometaphase (Dujardin et al., 1998; Coquelle et al., 2002; Tai et al., 2002;
Tanenbaum et al., 2006). The serine-rich regions encompassing the CAP-Gly domains of CLIP-170 also contribute to MT binding (Gupta et al., 2009, 2010). Recent studies have shown that phosphorylation(s) in the N-terminal serine-rich regions of CLIP-170 strengthens the aforementioned inhibitory intramolecular association (Lee et al., 2010) and is involved in its localization at kinetochores during mitosis ( $\mathrm{Li}$ et al., 2010). We previously demonstrated that AMP-activated protein kinase (AMPK) phosphorylates serine 312 (Ser312) in the third serine-rich region of CLIP-170 and that this phosphorylation is necessary for MT stabilization and adhesion turnover in polarized migrating cells (Nakano et al., 2010). However, how phosphorylation regulates CLIP-170 activity is largely unknown.

Here, we demonstrate the control of CLIP-170 activity by phosphorylation. With the benefit of an antiphosphorylated CLIP-170 antibody, we found that polo-like kinase1 (Plk1) mediates the transient increase of CLIP-170 phosphorylation at Ser312 during mitosis. This phosphorylation primarily affects the CLIP-170/MT interaction, independent of intermolecular associations or binding to EBs. In addition, RNAi studies showed that a proper phosphorylation and dephosphorylation cycle at Ser312 is required for the formation of stable kinetochore/MT attachments and subsequent chromosomal alignment in dividing cells. Thus, we propose the dynamic regulation of CLIP-170 by phosphorylation, which depends on the cellular context.

\section{Materials and Methods}

\section{Reagents and chemicals}

The anti-phosphorylated CLIP-170 (Ser312) antibody and the anti-CLIP-170 antibody were generated, purified, and characterized as described previously (Fukata et al., 2002; Nakano et al., 2010). The specificity of the anti-phosphorylated CLIP-170 (Ser312) antibody was further confirmed by CLIP-170 depletion (Fig. S1). The following commercial antibodies were used: antihistone H3 (Cell Signaling Technology, Danvers, MA, US), antiphosphorylated histone H3 (Ser10) (Cell Signaling Technology), anti-Plk1 (Cell Signaling Technology), mouse monoclonal antiCLIP-170 (Santa Cruz Biotechnology, Santa Cruz, CA, US), anti$\alpha$-tubulin (Sigma-Aldrich, St. Louis, MO, US or Millipore, Billerica, MA, US), and anti-GFP (Roche, Basel, Switzerland). Porcine brain tubulin and rhodamine-labeled tubulin were purchased from Cytoskeleton (Denver, CO, US). Other chemical reagents were obtained from commercial sources. We used the following siRNAs from Sigma-Aldrich: siCLIP-170 \#1, gacgaaaccuucugaugaa; siCLIP-170 \#2, gcauucaguuguucagacu; siPlk1 \#1, caacggcagcgugcagaucaa; and siPlk1 \#2, aagggcggcuuugccaagugc. The Plk1 siRNA sequences were described previously (Liu and Erikson, 2002). 


\section{Plasmid construction}

CLIP-170 cDNA was described previously (Fukata et al., 2002) and EB3 cDNA was obtained from Invitrogen (Grand Island, NY, US). Fragments of cDNA were amplified using PCR and subcloned into the pGEX (GE Healthcare, Buckinghamshire, UK), pRSET-c1, or pEGFP (Clontech Laboratories, Mountain View, CA, US) vectors. Point mutations of CLIP-170 were generated using PCR-based site-directed mutagenesis. For the rescue experiments, we utilized siRNA-insensitive CLIP-170 mutant generated by introducing silent mutations within the siRNA target sequence. All fragments were confirmed by DNA sequencing.

\section{Protein purification}

GST-fused +TIPs were produced in BL21(DE3) cells and purified on Glutathione Sepharose 4B beads (GE Healthcare) as described previously (Watanabe et al., 2009). His fusion proteins were produced in BL21(DE3) cells. The cells were lysed in buffer A (20 $\mathrm{mM}$ Tris- $\mathrm{HCl}$ [pH 7.5], $1 \mathrm{mM}$ EDTA, $1 \mathrm{mM}$ dithiothreitol [DTT], $300 \mathrm{mM} \mathrm{NaCl}$ ) containing $10 \mathrm{mM}$ imidazole and protease inhibitors, and the clarified lysates were loaded onto Ni-NTA columns (Qiagen, Venlo, Netherlands). After protein binding, the columns were washed with buffer A containing $30 \mathrm{mM}$ imidazole followed by elution with buffer A containing $300 \mathrm{mM}$ imidazole. For the in vitro end-tracking experiment, the affinity-purified proteins were further fractionated by size-exclusion chromatography with an ÄKTA purifier and Superdex 200 beads (GE Healthcare) equilibrated against buffer A. The peak fractions were used for the experiment and visualized with Coomassie brilliant blue staining, as shown in Fig. 5A. For the FLAG fusion proteins, pcDNA3.1FLAG-CLIP-170-full length (FL), -NT (1-602 aa), or - $\triangle 602$ was transfected into COS-7 cells. The cells were lysed in $20 \mathrm{mM}$ Tris$\mathrm{HCl}$ (pH 8.0), $1 \mathrm{mM}$ EDTA, $1 \mathrm{mM}$ DTT, $150 \mathrm{mM} \mathrm{NaCl}, 0.5 \%$ Triton X-100, $1 \mathrm{mM} \mathrm{Na}_{3} \mathrm{VO}_{4}, 100 \mathrm{mM} \mathrm{NaF}$, and protease inhibitors, and the clarified lysates were batch incubated overnight with anti-FLAG M2 affinity gel (Sigma-Aldrich). After protein binding, the resin was washed with buffer B $(20 \mathrm{mM}$ Tris- $\mathrm{HCl}[\mathrm{pH}$ 8.0], $1 \mathrm{mM}$ EDTA, $1 \mathrm{mM}$ DTT, $300 \mathrm{mM} \mathrm{NaCl}$ ) containing $0.25 \%$ Triton X-100. The proteins were eluted in buffer B containing 1.0 $\mathrm{mg} / \mathrm{ml} \mathrm{FLAG} \mathrm{and} 1.0 \mathrm{mg} / \mathrm{ml}$ 3xFLAG peptides.

\section{In vitro phosphorylation assay}

Purified mitotic kinases (GST-Aurora A, GST-Aurora B, GSTcyclin B1/cyclin-dependent kinase 1 (Cdk1), and GST-Plk1) were prepared as described previously (Goto et al., 2002; Yamaguchi et al., 2005; Shiromizu et al., 2006; Inoko et al., 2012). The kinase assay was performed with GST-Aurora A, GST-Aurora B, GSTcyclin B1/Cdk1, or GST-Plk1 in $20 \mu$ of kinase buffer C ( $25 \mathrm{mM}$

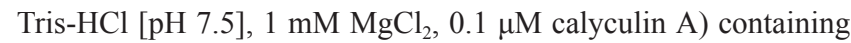
$40 \mathrm{ng} / \mu \mathrm{l}$ recombinant kinase and $2 \mu \mathrm{M}$ substrate (His-mGFPCLIP-170-NT wild type (WT) or A312) and $0.1 \mathrm{mM}\left[\gamma^{-32} \mathrm{P}\right]$ ATP. After incubation for $60 \mathrm{~min}$ at $30^{\circ} \mathrm{C}$, the reaction mixtures were boiled in SDS sample buffer and subjected to SDS-PAGE, fol- lowed by silver staining. The radiolabeled bands were visualized with an image analyzer (Typhoon FLA 9000; GE Healthcare). To prepare either phosphorylated or non-phosphorylated CLIP-170NT, the phosphorylation experiment with GST-Plk1 was carried out in $50 \mu \mathrm{l}$ or $20 \mu \mathrm{l}$ of kinase buffer D (25 mM Tris- $\mathrm{HCl}[\mathrm{pH}$ 7.5], $10 \mathrm{mM} \mathrm{MgCl}_{2}, 1 \mathrm{mM}$ EGTA, $1 \mathrm{mM}$ DTT, $0.1 \mu \mathrm{M}$ calyculin A) containing $60 \mathrm{ng} / \mu \mathrm{l}$ GST-Plk1 and $2 \mu \mathrm{M}$ His-mGFPCLIP-170-NT in the presence or absence of $0.1 \mathrm{mM}$ ATP at $30^{\circ} \mathrm{C}$ for $120 \mathrm{~min}$. The phosphorylation experiment with AMPK was performed in $50 \mu \mathrm{l}$ of kinase buffer E (40 mM Na-HEPES [pH 7.0], $80 \mathrm{mM} \mathrm{NaCl}, 5 \mathrm{mM} \mathrm{MgCl} 2,0.8 \mathrm{mM}$ EDTA, $0.8 \mathrm{mM}$ DTT, $0.2 \mathrm{mM}$ AMP, $8 \%$ glycerol) containing $0.1 \mathrm{ng} / \mu \mathrm{l}$ active AMPK ( $\alpha 1, \beta 1, \gamma 1)$ (Millipore), $2 \mu \mathrm{M}$ His-mGFP-CLIP-170-NT, and 0.2 $\mathrm{mM}$ ATP at $30^{\circ} \mathrm{C}$ for $60 \mathrm{~min}$.

\section{Cell culture and drug treatment}

HeLa cells were maintained in DMEM (Sigma-Aldrich) supplemented with 10\% fetal bovine serum (Nichirei Biosciences Inc., Tokyo, Japan). Plasmid transfection was performed with Lipofectamine (Invitrogen) according to the manufacturer's instructions. Lipofectamine RNAiMAX (Invitrogen) was used for siRNA transfection. Cell cycle synchronization was accomplished using a double thymidine block; cells were treated with $2.5 \mathrm{mM}$ thymidine (Sigma-Aldrich) for $17 \mathrm{~h}$, released for $9 \mathrm{~h}$, and then treated with thymidine again for $15 \mathrm{~h}$. To arrest the cell cycle at prometaphase, cells were treated with $7.5 \mu \mathrm{M}$ S-trityl-L-cysteine (STLC) or $100 \mathrm{ng} / \mathrm{ml}$ nocodazole for $16 \mathrm{~h}$. To inhibit Plk1, cells were treated for $1 \mathrm{~h}$ before lysis with $100 \mathrm{nM}$ BI 2536 (Chemie Tek, Indianapolis, IN, US).

\section{MT cosedimentation assay}

The cosedimentation assay was performed as previously described (Folker et al., 2005) with some modifications. Phosphorylated and non-phosphorylated mGFP-CLIP-170-NT were prepared, and 0.5 $\mu \mathrm{M}$ of each protein was incubated with increasing amounts of taxol-stabilized MTs in PEM buffer (100 mM K-PIPES [pH 6.9], $1 \mathrm{mM}$ EGTA, $1 \mathrm{mM} \mathrm{MgSO}$ ) at $37^{\circ} \mathrm{C}$ for $20 \mathrm{~min}$. The samples were centrifuged at $65,000 \mathrm{~g}$ for $30 \mathrm{~min}$ at $37^{\circ} \mathrm{C}$. The supernatant and pellet were collected and analyzed by SDS-PAGE.

\section{Turbidity measurements}

Phosphorylated or non-phosphorylated mGFP-CLIP-170-NT was mixed with $10 \mu \mathrm{M}$ tubulin in assembly buffer (40 mM K-HEPES [pH 7.0], $40 \mathrm{mM} \mathrm{NaCl}, 1 \mathrm{mM}$ EGTA, $5 \mathrm{mM} \mathrm{MgCl}$, $5 \%$ DMSO, $2 \mathrm{mM}$ GTP, 4\% glycerol) and then injected into a quartz cuvette. The absorbance at $350 \mathrm{~nm}$ was monitored at $15.6 \mathrm{sec}$ intervals at $37^{\circ} \mathrm{C}$ using a DU800 spectrophotometer (Beckman Coulter, Brea, $\mathrm{CA}$, US). The initial $\mathrm{A}_{350}$ value was utilized for baseline adjustment.

\section{In vitro binding assay}

mGFP-CLIP-170-NT was subjected to a kinase assay with Plk1 in 
the presence or absence of ATP and then mixed with GST-EB3immobilized affinity beads in assay buffer $(25 \mathrm{mM}$ Tris- $\mathrm{HCl}[\mathrm{pH}$ 7.5], $100 \mathrm{mM} \mathrm{NaCl}, 10 \mathrm{mM} \mathrm{MgCl}, 1 \mathrm{mM}$ EGTA, $1 \mathrm{mM}$ DTT, $0.1 \mu \mathrm{M}$ calyculin $\mathrm{A}, 0.25 \%$ Brij-35). The beads were then washed with assay buffer, and the bound proteins were subjected to SDSPAGE, followed by Coomassie brilliant blue staining.

\section{In vitro tracking of CLIP-170}

Reconstitution of end-tracking was performed according to previous reports (Gell et al., 2010; Li et al., 2011). The indicated concentration of mGFP-CLIP-170-NT was incubated with $15 \mu \mathrm{M}$ tubulin (containing $0.5 \mu \mathrm{M}$ rhodamine-tubulin) and $400 \mathrm{nM}$ EB3. In vitro tracking experiments with CLASP2 were performed with $15 \mu \mathrm{M}$ tubulin (containing $0.5 \mu \mathrm{M}$ rhodamine-tubulin), $400 \mathrm{nM}$ EB3, $100 \mathrm{nM}$ His-CLIP-170-NT, and $25 \mathrm{nM}$ or $10 \mathrm{nM}$ HismGFP-CLASP2. During the experiments, the samples were kept at $25 \pm 1{ }^{\circ} \mathrm{C}$. Images were collected every $3 \mathrm{sec}$ using a total internal reflection fluorescence microscope (TE2000-E; Nikon, Tokyo, Japan), an EM charge-coupled device camera (iXonEM+ 897; Andor, South Windsor, CT, US), and a 488/561-nm excitation laser. We used a dual bandpass dichroic mirror (51004vs2bs; Chroma Technology, Rockingham, VT, US) and the FF01-520/35 and FF01-593/40 emission filters (Semrock, Rochester, NY, US). Data analysis was performed with ImageJ (http://rsb.info.nih.gov/ $\mathrm{ij} /)$.

\section{Immunofluorescent staining}

Cells were fixed with methanol containing $1 \mathrm{mM}$ EGTA at $-30^{\circ} \mathrm{C}$ for 10 min and postfixed with $4 \%$ formaldehyde in PBS at room temperature for $10 \mathrm{~min}$, followed by permeabilization with $0.15 \%$ Triton X-100 at room temperature for an additional $5 \mathrm{~min}$. After blocking with $1 \mathrm{mg} / \mathrm{ml} \mathrm{BSA} \mathrm{(Sigma-Aldrich)} \mathrm{in} \mathrm{PBS} \mathrm{for} 30 \mathrm{~min}$ at room temperature, the cells were incubated with the indicated primary and secondary antibodies at room temperature for $1 \mathrm{~h}$, respectively. For kinetochore staining, the cells were fixed with $3 \%$ paraformaldehyde and $2 \%$ sucrose in PBS at room temperature for $10 \mathrm{~min}$, permeabilized with $0.5 \%$ Triton X-100 on ice for 5 min, and then blocked with $10 \%$ donkey serum in PBS at room temperature for $30 \mathrm{~min}$. The cells were incubated with the indicated primary antibodies diluted in PBS at $37^{\circ} \mathrm{C}$ for $1 \mathrm{~h}$. The cells were observed using a 1.4 NA CFI Plan-Apo VC 60X or PlanApo 63X oil immersion objective lens under a confocal laser microscope (A1Rsi; Nikon or LSM780; Carl Zeiss, Jena, Germany). All presented images are maximum-intensity projections of confocal sections (thickness of $0.4-0.8 \mu \mathrm{m}$ ). To evaluate chromosome alignment, we counted the number of cells with unaligned chromosomes among the cells displaying bipolar spindles (Wong and Fang, 2006). Cold treatment and measurement of spindle fluorescent intensity were performed according to a previous report (DeLuca, 2010). Spindle intensities were quantified using ImageJ.

\section{Statistical analysis}

Statistical analyses were performed with GraphPad Prism version 5.0 (GraphPad Software, La Jolla, CA, US). $\mathrm{P}<0.05$ was considered statistically significant.

\section{Results}

\section{CLIP-170 phosphorylation at Ser312 is increased during mitosis}

+TIP activity must be tightly controlled during the cell cycle for the dynamic reorganization of the MT cytoskeleton. Recent reports have shown that the phosphorylation of Ser312 in the third serine-rich region of CLIP-170 plays a key role in regulating CLIP-170 activity (Lee et al., 2010; Nakano et al., 2010). To monitor the dynamic changes in CLIP-170 activity throughout the cell cycle, we utilized an antibody that specifically recognizes CLIP-170 phosphorylated at Ser312 (Fig. 1A). HeLa cells were synchronized with a double thymidine block and lysed after the final release, followed by immunoblot analysis with antiphosphorylated CLIP-170 (Ser312) and histone H3 (Ser10) antibodies. Histone $\mathrm{H} 3$ phosphorylation was predominantly detected at $10 \mathrm{~h}$ after the final release, confirming the increase in mitotic cells at that time point (Fig. 1B). Under this condition, CLIP-170 phosphorylation at Ser312 increased in a similar time-course to that of histone $\mathrm{H} 3$ phosphorylation that is prominent in prophase to anaphase (Goto et al., 2002). However, after the transient peak in histone H3 phosphorylation, the CLIP-170 phosphorylation increased further (12 h after release) and then decreased to the basal level (Fig. 1B). These results indicate that the phosphorylation level of CLIP-170 is dynamically and stringently controlled during cell cycle progression and that the serine 312 residue of CLIP-170 is predominantly phosphorylated during mitosis.

\section{Plk1 phosphorylates CLIP-170 at Ser312 in vitro}

Several kinases, including Plk1, Aurora A/B, and Cdk1, are activated during mitosis and are required for cell cycle progression. We first tested whether these kinases can phosphorylate CLIP-170 in vitro. We employed the N-terminal fragment of CLIP-170 (1-602 aa; CLIP-170-NT), which contains Ser312, as a fusion protein with a His tag and GFP at its N-terminus (Fig. 1A). When CLIP-170-NT WT was incubated with the candidate mitotic kinases, a radioactive band of CLIP-170-NT was evident with Cdk1 and Plk1 (Fig. 2A). The mutation of Ser312 to alanine markedly reduced CLIP-170 phosphorylation by Plk1 but not by Cdk1 (Fig. 2A), indicating that Cdk1 phosphorylates other residue(s), including Thr287 as previously reported (Yang et al., 2009). Immunoblot analysis with the anti- 
A

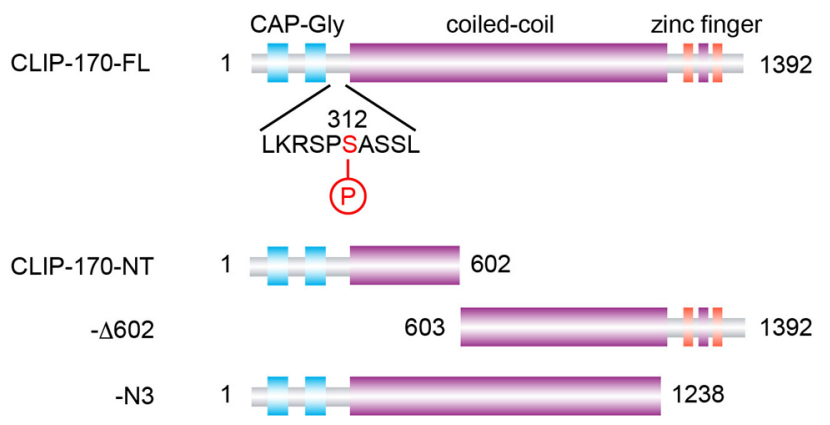

B
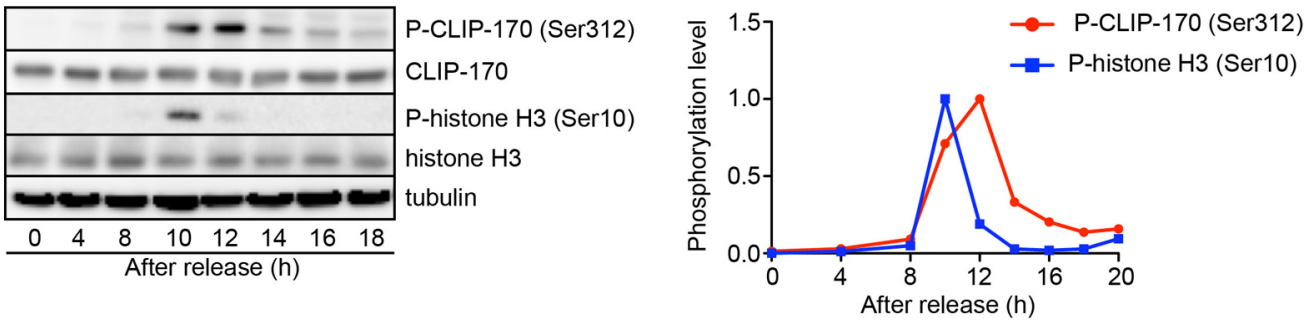

Fig. 1. Cell cycle progression transiently increases CLIP-170 phosphorylation at Ser312. (A) Schematic diagram of CLIP-170. The domain organization of CLIP-170 and its fragments is represented. The CLIP-170-NT fragment encompasses residues 1-602 from the N-terminus of CLIP-170, whereas $\Delta 602$ lacks the NT region. CLIP-170-NT contains Ser312 in the third serine-rich stretch. CLIP-170-N3 lacks the C-terminal zinc finger motifs. (B) HeLa cells were synchronized with a double thymidine block (see Materials and Methods). At the indicated time points after the final release, the cells were lysed and subjected to immunoblot analysis with the indicated antibodies. P-CLIP-170, phosphorylated CLIP-170 (Ser312). P-histone H3, phosphorylated histone H3 (Ser10). The plots on the right show the quantified data from the immunoblot (left) of phosphorylated CLIP-170 (Ser312) and phosphorylated histone H3 (Ser10). Phosphorylation was transiently increased 10-12 h after the release. The results are representative of at least four experiments.

phosphorylated CLIP-170 antibody further demonstrated that Plk1 indeed phosphorylated Ser312 (Fig. 2B). Next, we examined whether Plk1 phosphorylates other regions of CLIP-170 than the N-terminal regions using FLAG-tagged CLIP-170-FL, -NT, and - $\Delta 602$ (Fig. 1A). Under conditions in which the phosphorylation of CLIP-170-NT by Plk1 was obvious, CLIP-170- $\Delta 602$ was only minimally phosphorylated (Fig. 2C), suggesting that Plk1 mainly phosphorylates the N-terminal region (1-602 aa) of CLIP-170. An alanine mutation at Ser195, which was previously identified as a Plk1-mediated phosphorylation site (Li et al., 2010), only slightly diminished the phosphorylation by Plk1 (Fig. 2D). Furthermore, the double alanine mutant A195/A312 was still phosphorylated by Plk1 to a level similar to that of the single A312 mutant, indicating that a residue(s) other than Ser195 and Ser312 contributes to the phosphorylation mediated by Plk1. Together, the results from our in vitro phosphorylation analysis indicate that Plk1 phosphorylates CLIP-170 at Ser312.

\section{Plk1 is responsible for the phosphorylation of CLIP-170 at Ser312 during mitosis}

To determine whether Plk1 phosphorylates CLIP-170 at Ser312 in mitotic cells, we examined the effects of the pharmacological inhibitor on CLIP-170 Ser312 phosphorylation during mitosis. Synchronized HeLa cells were treated with the Plk1 inhibitor BI2536 for $1 \mathrm{~h}$ before they were lysed (Fig. 3A). CLIP-170 phosphorylation at Ser312 was increased at 10 and $12 \mathrm{~h}$ after the final thymidine block release (Fig. 1B and Fig. 3A). Treatment with the Plk1 inhibitor BI2536 diminished this increase in CLIP-170 phosphorylation (Fig. 3A). An RNAi approach was also employed to test the involvement of Plk1 in CLIP-170 phosphorylation. Plk1 depletion from synchronized HeLa cells resulted in a large reduction in CLIP-170 phosphorylation at Ser312 (Fig. 3B). Another siRNA targeting Plk1 yielded essentially similar results (data not shown). Of note, consistent with the crucial role of this mitotic kinase in mitotic entry (Macurek et al., 2008; Seki et al., 2008), both inhibitor treatment and Plk1 depletion also affected the phosphorylation level of histone H3 (Fig. 3A and B). 
A

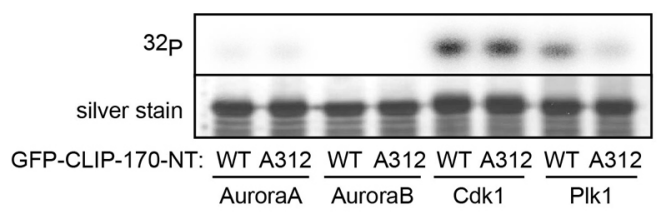

B

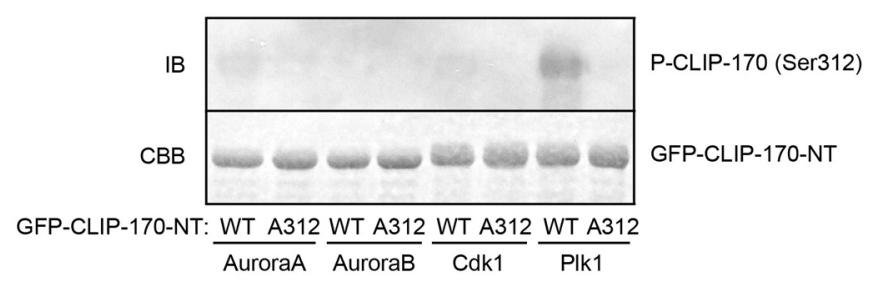

C

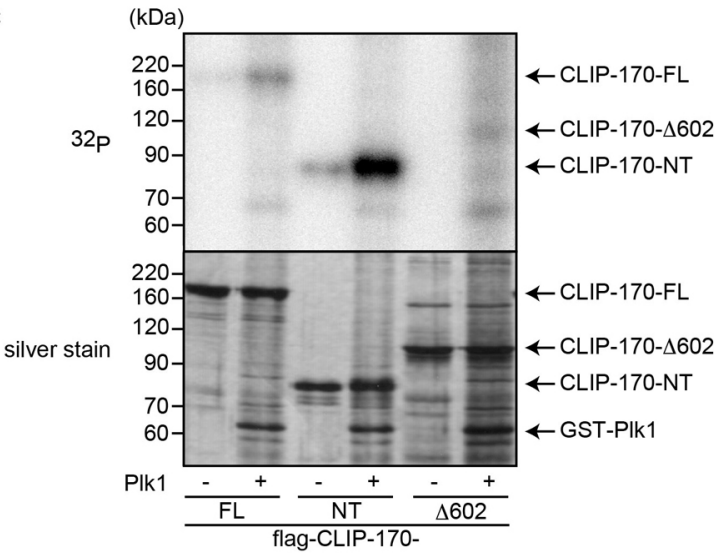

D
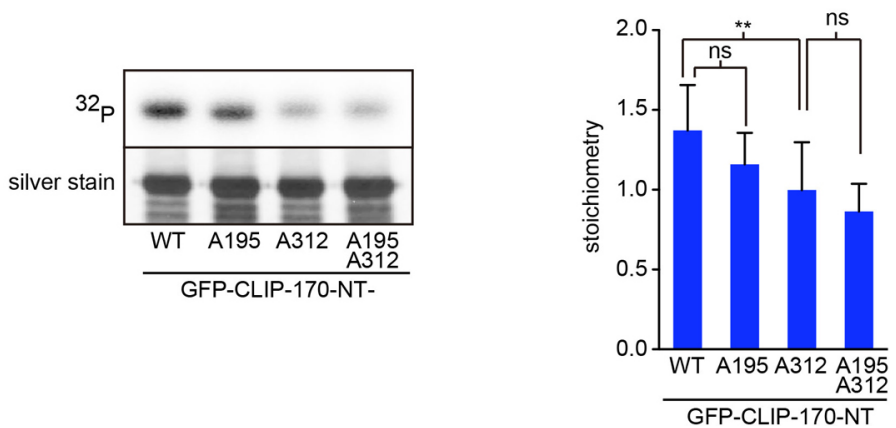

Fig. 2. Plk1 phosphorylates CLIP-170 at Ser312 in vitro. (A) Purified GFP-CLIP-170-NT (presented in Fig. 1) was incubated with the indicated mitotic kinases in the presence of radiolabeled ATP, followed by silver staining (lower panel) and autoradiography (upper panel). Cdk1 and Plk1 strongly phosphorylated CLIP-170-NT. Alanine mutation at Ser312 significantly decreased the phosphorylation by Plk1. (B) CLIP-170-NT was incubated with the indicated kinases and subjected to immunoblotting with the anti-phosphorylated CLIP-170 (Ser312) antibody. (C) Purified CLIP-170-FL, -NT, or - $\Delta 602$ were incubated with or without Plk1. CLIP-170 phosphorylation was visualized by autoradiography. Plk1 predominantly phosphorylated the N-terminal region of CLIP-170. (D) Mutational analysis of CLIP-170 phosphorylation by Plk1. CLIP-170-NT WT or its mutants were incubated with Plk1. CLIP-170 phosphorylation was visualized by autoradiography. Mutation at Ser312 resulted in a decrease in Plk1-mediated phosphorylation. The bar graph on the right shows the phosphorylation stoichiometry. The data represent the means $\pm \mathrm{SD}$. ${ }^{* *} \mathrm{p}<0.01$; ns, not significant (one-way repeated-measures ANOVA, Tukey's HSD). The results are representative of at least three experiments. 
When HeLa cells were synchronized to prometaphase by the treatment with an Eg5 inhibitor STLC, in which the phosphorylation of histone $\mathrm{H} 3$ was minimally affected, the Plk1 inhibitor partially reduced the level of CLIP-170 phosphorylation at Ser312 (Fig. 3C). By analyzing these data together with the in vitro results (Fig. 2), we conclude that Plk1 mediates the phosphorylation of CLIP-170 at Ser312 at prometaphase during mitosis.

\section{Phosphorylation of CLIP-170 at Ser312 inhibits its binding to MTs and suppresses its ability to facilitate MT nucleation/polymerization}

The phosphorylation of CLIP-170 at Ser312 alters its accumulation at MT ends in cells (Fig. S2; Lee et al., 2010; Nakano et al., 2010). We therefore addressed the effects of CLIP-170 phosphorylation on its binding to MTs by biochemical methods using purified proteins. A cosedimentation assay with various amounts of taxol-stabilized MTs showed that phosphorylated CLIP-170-NT bound to MTs at a lower level than non-phosphorylated CLIP-170, especially at a low MT concentration (Fig. 4A). Conversely, even at a high MT concentration, phosphorylated CLIP170-NT was detectable in the supernatant fraction, while non-phosphorylated CLIP-170 was not (Fig. 4A). Quantification of the bound CLIP-170 fraction demonstrated the inhibitory effects of CLIP-170 phosphorylation on its association with MTs (Fig. 4A). We also performed the same experiment using AMPK, which has been shown to phosphorylate Ser312 in CLIP-170 (Nakano et al., 2010), and obtained similar results (Fig. 4B), indicating that the phosphorylation of CLIP-170 at Ser312 is sufficient to decrease its binding to MTs. However, we could not completely exclude the possible involvement of other site(s) in the effects of Plk1-mediated phosphorylation. When we monitored MT nucleation, polymerization, and bundling in the presence of CLIP-170 by turbidity, consistent with the binding of CLIP-170 to MTs, non-phosphorylated CLIP170 more efficiently induced turbidity than phosphorylated CLIP-170, and this increase in turbidity was time dependent (Fig. 4C). This difference was observed at two CLIP170 concentrations $(0.5$ and $0.3 \mu \mathrm{M}$, Fig. $4 \mathrm{C})$. Furthermore, we examined the effects of CLIP-170 phosphorylation on binding to EB3 by a pull-down experiment, but did not find any detectable differences (Fig. 4D). In addition, phosphorylation of CLIP-170 by Plk1 did not affect the interaction between its $\mathrm{N}$ - and C-terminal regions (Fig. S3A). Thus, the results of our experiments using purified proteins indicate that CLIP-170 phosphorylation at Ser312 primarily decreases its binding to MTs and its ability to nucleate/ polymerize MTs, independent of EBs and its intramolecular association.
A

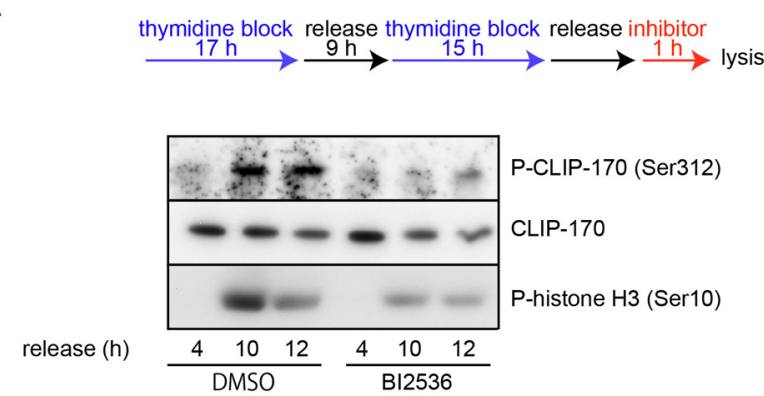

B
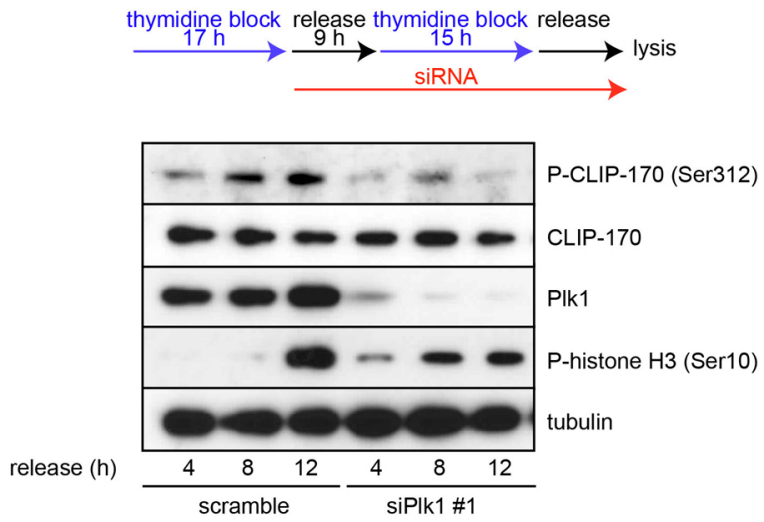

C

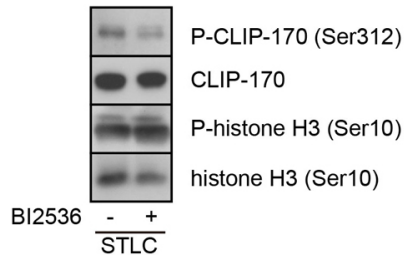

Fig. 3. Plk1 is responsible for CLIP- 170 phosphorylation at Ser 312 during mitosis. (A) Synchronized HeLa cells were treated with vehicle DMSO or the Plk1 inhibitor BI2536 $(100 \mathrm{nM})$ for $1 \mathrm{~h}$ before lysis. The treated lysates were subjected to immunoblot analysis with the indicated antibodies. Plk1 inhibition impaired CLIP-170 phosphorylation at Ser312. (B) HeLa cells were transfected with either control scramble or Plk1 siRNA during the double thymidine block. The cells were lysed at the indicated time points after release and subjected to immunoblot analysis. Phosphorylation of CLIP-170 at Ser312 was decreased in the Plk1depleted cells. The results are representative of at least four experiments. (C) HeLa cells were treated with Eg5 inhibitor STLC $(7.5 \mu \mathrm{M})$ for $16 \mathrm{~h}$, and then incubated with or without Plk1 inhibitor (100 nM) for additional $1 \mathrm{~h}$. The lysates were subjected to immunoblot analysis with the indicated antibodies.

\section{CLIP-170 phosphorylation impairs its end-tracking ability}

Experiments using purified proteins and total internal reflection fluorescent microscopy allowed us to reconstitute the dynamic behavior of +TIPs and to measure the dynamics of individual MTs in vitro (Gell et al., 2010; Telley et 
A
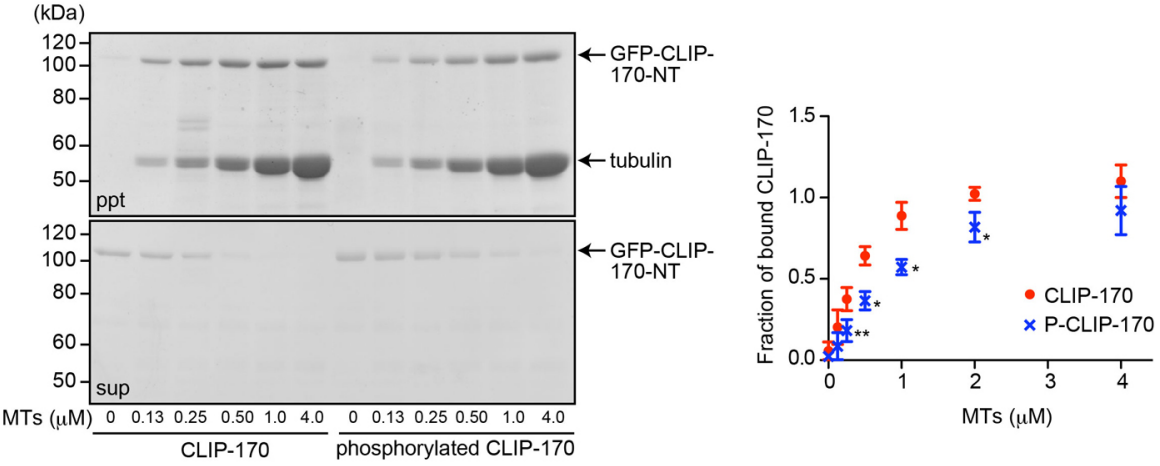

B $\quad(\mathrm{kDa})$
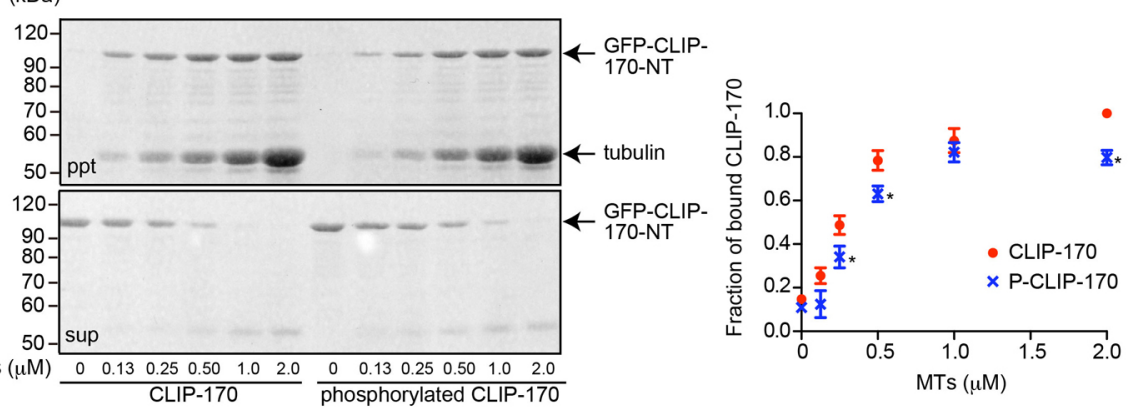

C

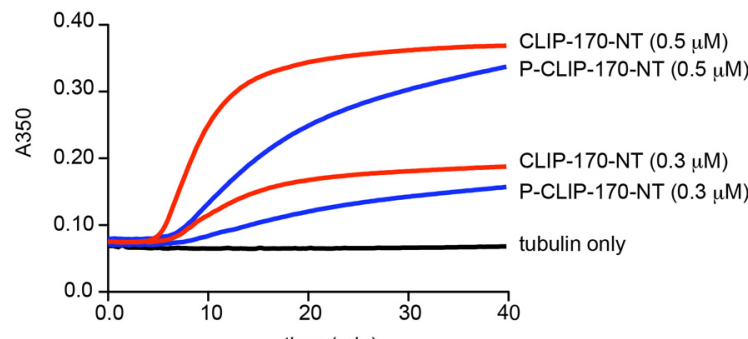

D
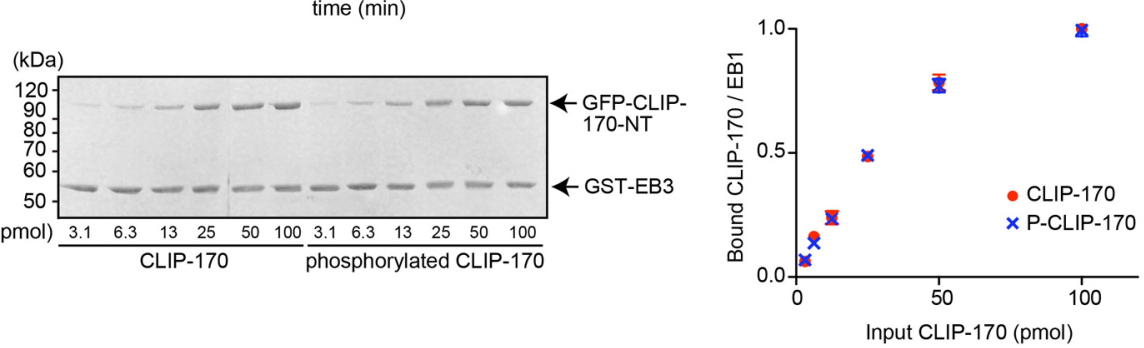

Fig. 4. Phosphorylation of CLIP-170 at Ser312 inhibits its binding to MTs and suppresses its ability to facilitate MT nucleation/polymerization. (A) Cosedimentation assay between taxol-stabilized MTs and GFP-CLIP-170-NT. Increasing amounts of MTs were incubated with a fixed concentration of GFP-CLIP-170-NT. Purified GFP-CLIP-170-NT was incubated with Plk1 in the presence or absence of ATP prior to the assay. The pellet (ppt) and supernatant (sup) fractions were stained with Coomassie brilliant blue and are shown in the upper panel and lower panel, respectively. The plot (right) shows the quantitative data from the gel. The phosphorylation of CLIP-170 significantly reduced its binding to MTs. The data represent the means \pm SD. ${ }^{*} \mathrm{p}<0.05, * * \mathrm{p}<0.01$ versus non-phosphorylated CLIP-170 at the same MT concentration (t-test). (B) Cosedimentation assay between taxol-stabilized MTs and GFP-CLIP-170-NT. AMPK was used to phosphorylate CLIP-170 instead of Plk1. ${ }^{*} \mathrm{p}<0.05$ versus non-phosphorylated CLIP-170 at the same MT concentration (t-test). (C) MT nucleation/polymerization in the presence of phosphorylated or non-phosphorylated CLIP-170. The absorbance at $350 \mathrm{~nm}$ was monitored as the readout of MT polymerization. (D) Binding of phosphorylated or non-phosphorylated CLIP-170 to EB3. Beads coated with GSTEB3 were incubated with increasing amounts of GFP-CLIP-170-NT. Bound CLIP-170 was analyzed by Coomassie brilliant blue staining. No significant difference was observed between phosphorylated and non-phosphorylated CLIP-170. The data represent the means $\pm \mathrm{SD}$, and the results are representative of at least four experiments. 
CLIP-170 Phosphorylation

A

His-GFP-CLIP-170-NT

His-EB3

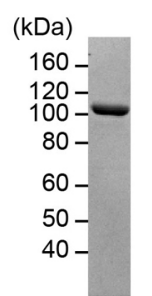

$(\mathrm{kDa})$

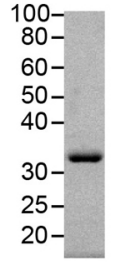

B

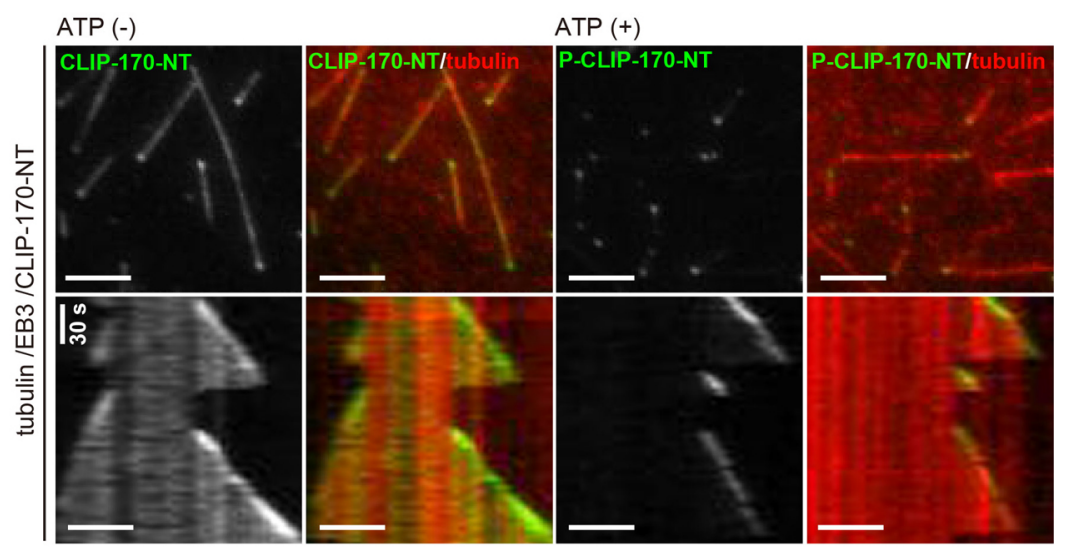

End intensity

Lattice intensity
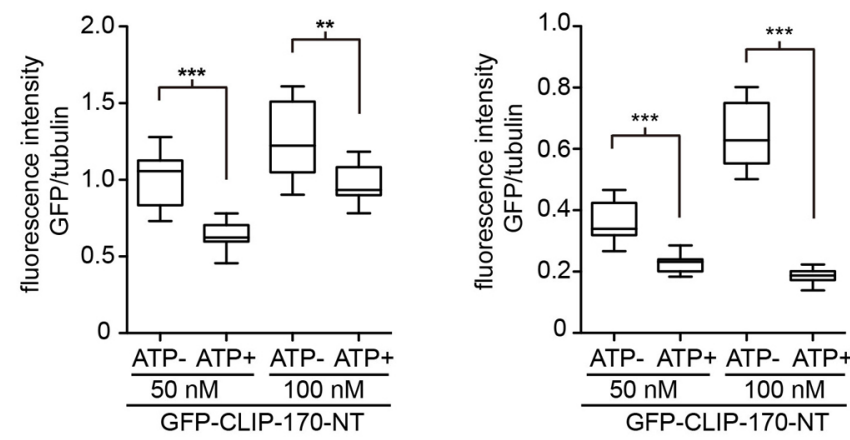

C

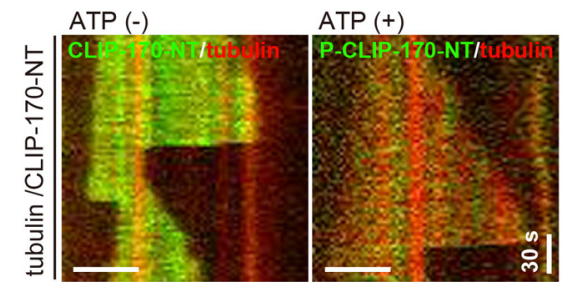

Fig. 5. CLIP-170 phosphorylation impairs its end-tracking ability. (A) Coomassie brilliant blue staining of the purified proteins used for end-tracking in vitro (GFP-CLIP-170-NT and His-EB3). (B) Images (top) and kymographs (bottom) of the end-tracking of phosphorylated or non-phosphorylated GFPCLIP-170-NT $(50 \mathrm{nM})$ in the presence of rhodamine-labeled tubulin $(15 \mu \mathrm{M}$ total tubulin) and EB3 (400 nM). The fluorescence intensity of GFPCLIP-170-NT at the ends and lattice of each MT was quantified and is shown as a ratio to tubulin intensity (lower graphs). Phosphorylated CLIP-170 displayed decreased accumulation at both the MT ends and lattice. A high concentration of GFP-CLIP-170-NT (100 nM) yielded similar results. The data represent the means $\pm \mathrm{SD}$ of more than three independent experiments. $\mathrm{n}>50 .{ }^{* *} \mathrm{p}<0.01,{ }^{* * *} \mathrm{p}<0.001$ versus the respective control (t-test with Welch's correction). (C) Kymographs of MT with phosphorylated or non-phosphorylated CLIP-170 in the absence of EB3. Non-phosphorylated CLIP-170 was detected along the MTs to a greater degree than phosphorylated CLIP-170. The results are representative of more than three experiments. Bars, $3 \mu \mathrm{m}$. 
al., 2011). We examined the effects of Plk1-mediated CLIP-170 phosphorylation on its end-tracking activity. Because EBs (EB1 or EB3) are absolutely required for the end-tracking of CLIP-170 in vitro (Bieling et al., 2008; Dixit et al., 2009), EB3-FL was used in this experiment. Affinity-purified GFP-CLIP-170-NT and EB3 were further subjected to size-exclusion chromatography (see Materials and Methods for details). These proteins were visualized as single bands at the expected size by Coomasie brilliant blue staining (Fig. 5A). Purified GFP-CLIP-170-NT was incubated with Plk1 in the presence or absence of ATP and then used in the reconstitution experiment. Non-phosphorylated GFP-CLIP-170-NT primarily accumulated at the growing MT ends in the presence of EB3 and also showed some localization to the lattice (Fig. 5B). Under these conditions, the phosphorylation of CLIP-170 by Plk1 reduced its localization to the MT lattice to an undetectable level and partially diminished its accumulation at the ends (Fig. 5B). The quantification of the GFP-CLIP-170-NT fluorescence intensity demonstrated that phosphorylation significantly decreased the CLIP-170 intensity at both the MT ends and lattice. A larger decrease was observed at the lattice at the tested CLIP-170 concentrations $(50 \mathrm{nM}$ and $100 \mathrm{nM}$, Fig. 5B). Even in the absence of EB3, non-phosphorylated GFPCLIP-170-NT localized along the MTs without apparent accumulation at the ends. On the other hand, phosphorylated GFP-CLIP-170-NT was not as frequently found along the MTs (Fig. 5C). The results in Fig. 5 were essentially reproduced with AMPK instead of Plk1 (data not shown). Taken together with the previous results (Fig. 4), these data show that CLIP-170 phosphorylation by Plk1 primarily inhibits its binding to MTs.

\section{Phosphorylation of CLIP-170 at Ser312 is required for the formation of stable kinetochore/MT attachments and subsequent chromosome alignment}

CLIP-170 accumulates at the kinetochore during prometaphase to facilitate precise chromosome alignment (Dujardin et al., 1998; Tanenbaum et al., 2006). Considering the above observation that the mitotic kinase Plk1 controls CLIP-170 activity through phosphorylation, we therefore examined whether CLIP-170 phosphorylation at Ser312 is involved in proper chromosome alignment by RNA interference. The effectiveness of CLIP-170 siRNA was confirmed by immunoblot analysis. More than $90 \%$ of CLIP-170 was successfully depleted by siRNA transfection (Fig. 6A). Consistent with previous reports (Tanenbaum et al., 2006), misaligned chromosomes were frequently observed in the cells transfected with CLIP-170 siRNA (Fig. 6B). Approximately 55\% of the bipolar spindles in the CLIP-170-depleted cells displayed misaligned chromosomes, whereas only $20 \%$ of the bipolar spindles in the control cells did so (Fig. 6D). In the CLIP-170-depleted background, cells expressing an siRNA-resistant full-length
CLIP-170 partially rescued the chromosomal misalignment (recovered to $30 \%$, Fig. 6C and D). As expected, the expression of CLIP-170-N3 (Fig. 1A), which is defective in kinetochore localization (Dujardin et al., 1998), failed to rescue the chromosomal misalignment (Fig. 6D). Under these conditions, although mutation at Ser312 did not affect CLIP-170 accumulation at the kinetochores visualized by a kinetochore marker Hec1 (Fig. 6E), the expression of either non-phosphorylatable (A312) or phosphomimetic mutants (D312 or E312) could not rescue the inhibitory effects of CLIP-170 depletion (Fig. 6C and D). The proportion of misaligned chromosomes observed in the cells expressing these mutants was similar to that of the CLIP-170-depleted cells (approximately $60 \%$ in A312 and 50\% in D312 and E312, Fig. 6D). Thus, CLIP-170 phosphorylation at Ser312 appears to control chromosomal alignment independent of its kinetochore-targeting mechanism.

The alignment of the chromosomes at the metaphase plate requires the proper attachment of the MTs to the kinetochores and the formation of kinetochore MTs (kMTs) (Tanaka, 2010). Thus, finally, we investigated the involvement of CLIP-170 phosphorylation in these processes by measuring the amount of cold-stable MTs; kMTs are selectively stable against cold treatment, whereas cold treatment induces the depolymerization of non-kMTs (DeLuca, 2010). For this experiment, HeLa cells were treated as depicted in Fig. 7A and a previously reported method (DeLuca, 2010). Without cold treatment (0 min), neither CLIP-170 depletion nor the expression of CLIP-170 mutants affected the fluorescent intensity of the spindle (Fig. 7B left). When the control cells were treated at $4^{\circ} \mathrm{C}$ for $15 \mathrm{~min}$, robust spindles with cold-stable kMTs were observed (Fig. 7B right). In the CLIP-170-depleted cells, however, only a few cold-stable MTs remained. The fluorescence intensity of the spindles in the CLIP-170-depleted cells was reduced to approximately $50 \%$ of that observed in the control cells (Fig. 7B right). Under these conditions, the defect in the formation of cold-stable kMTs was rescued by CLIP-170 WT but not by A312 or E312 (Fig. 7B right). These results indicate that CLIP-170 and its precise phosphorylation/dephosphorylation cycle are required for the formation of stable kinetochore/MT attachments.

\section{Discussion}

\section{CLIP-170 phosphorylation and its kinases}

CLIP-170 was originally found to associate with MTs in a manner that is dependent on its phosphorylation state (Rickard and Kreis, 1990, 1991; Pierre et al., 1992). Recent studies have demonstrated that CLIP-170 is indeed phosphorylated and successfully identified the responsible kinases (Yang et al., 2009; Lee et al., 2010; Li et al., 2010; Nakano et al., 2010). We previously found that AMPK 
A

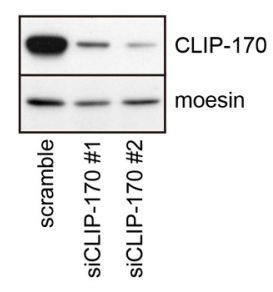

B

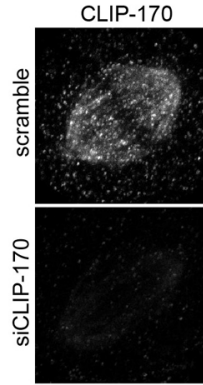

tubulin

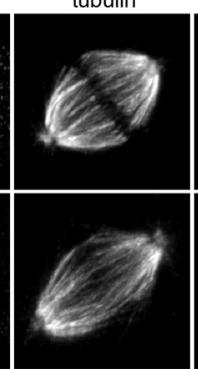

DNA CLIP-170/tubulin/DNA

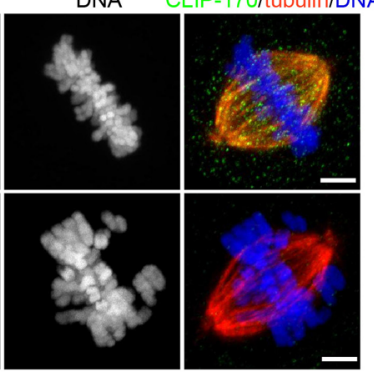

C

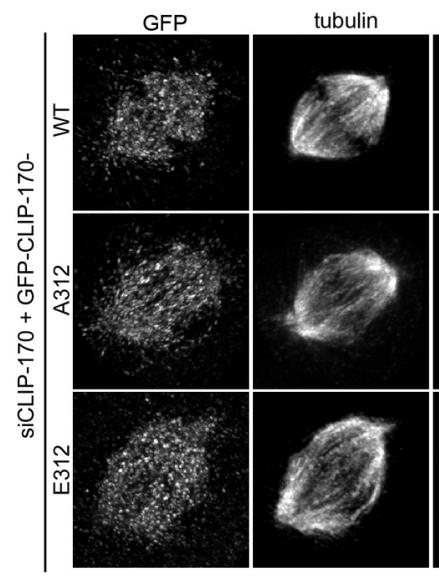

DNA

GFP/tubulin/DNA

D
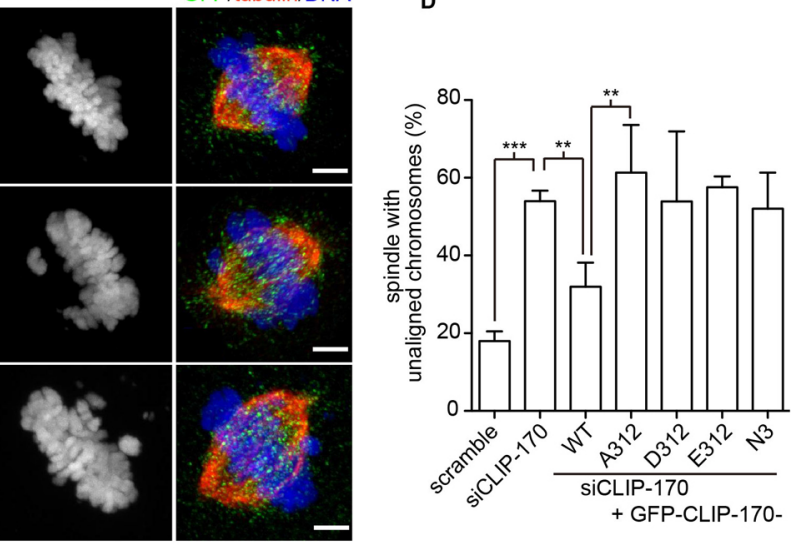

$\mathbf{E}$
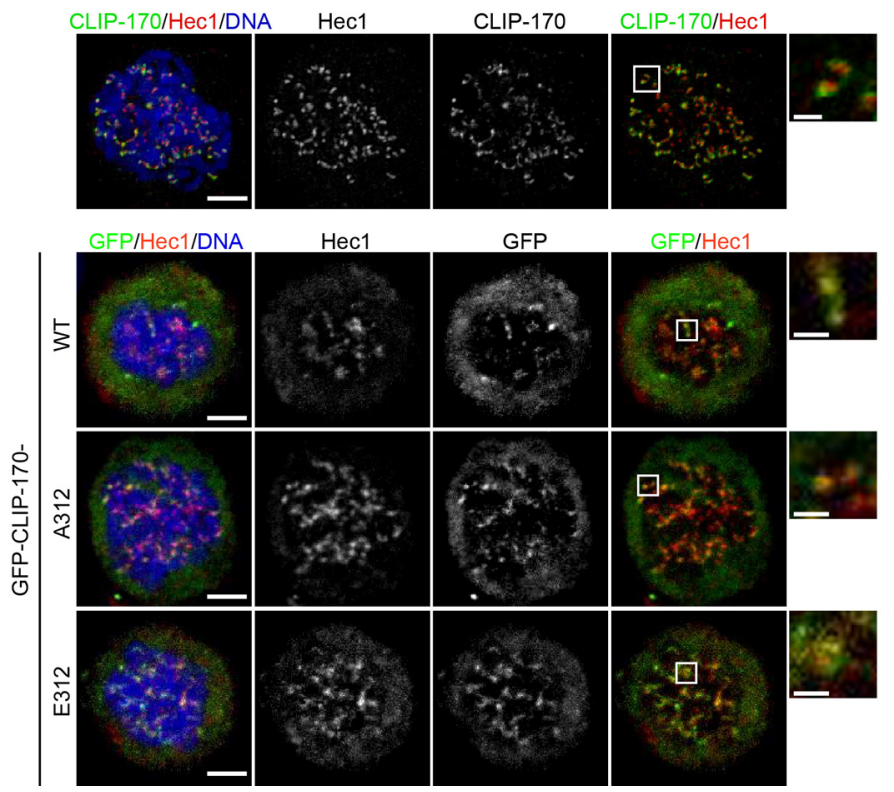

Fig. 6. Phosphorylation of CLIP-170 at Ser312 is required for chromosome alignment. (A) Immunoblot of HeLa cell lysates transfected with the indicated siRNAs. Moesin was used as the control protein. (B) HeLa cells were transfected with control scramble or CLIP-170 siRNA, cultured for $48 \mathrm{~h}$, and then stained with the indicated antibodies and DAPI. Depletion of CLIP-170 induced chromosomal misalignment. Bars, $5 \mu \mathrm{m}$. (C) Rescue of CLIP-170 depletion by the expression of CLIP-170 WT or its mutants. CLIP-170-depleted HeLa cells were transfected with the CLIP-170 constructs and then stained with the indicated antibodies and DAPI. The expression of CLIP-170 WT rescued the defects in chromosome alignment, but the expression of either the non-phosphorylatable or phosphomimetic mutant failed to do so. Bars, $5 \mu \mathrm{m}$. (D) The graph shows the quantitative data for the spindles with unaligned chromosomes. The data represent the means $\pm \mathrm{SD}$ of more than three independent experiments. $\mathrm{n}>100 . * * \mathrm{p}<0.01, * * * \mathrm{p}<0.001$ (one-way repeated-measures ANOVA, Tukey's HSD). (E) HeLa cells were treated with $100 \mathrm{ng} / \mathrm{ml}$ nocodazole for $16 \mathrm{~h}$ and stained with the indicated antibodies. CLIP-170 or its mutants were detected at Hec1-containing kinetochores. Representative kinetochores (insets) are magnified and shown in the right panels. Bars in left and magnified panels, 5 and $1 \mu \mathrm{m}$, respectively. The results are representative of at least four experiments. 
A

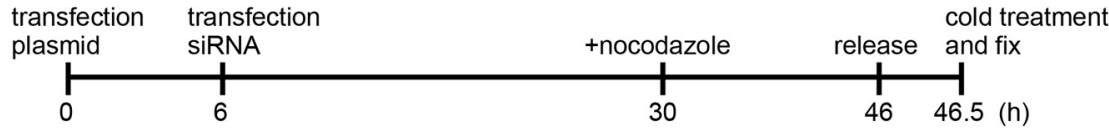

B
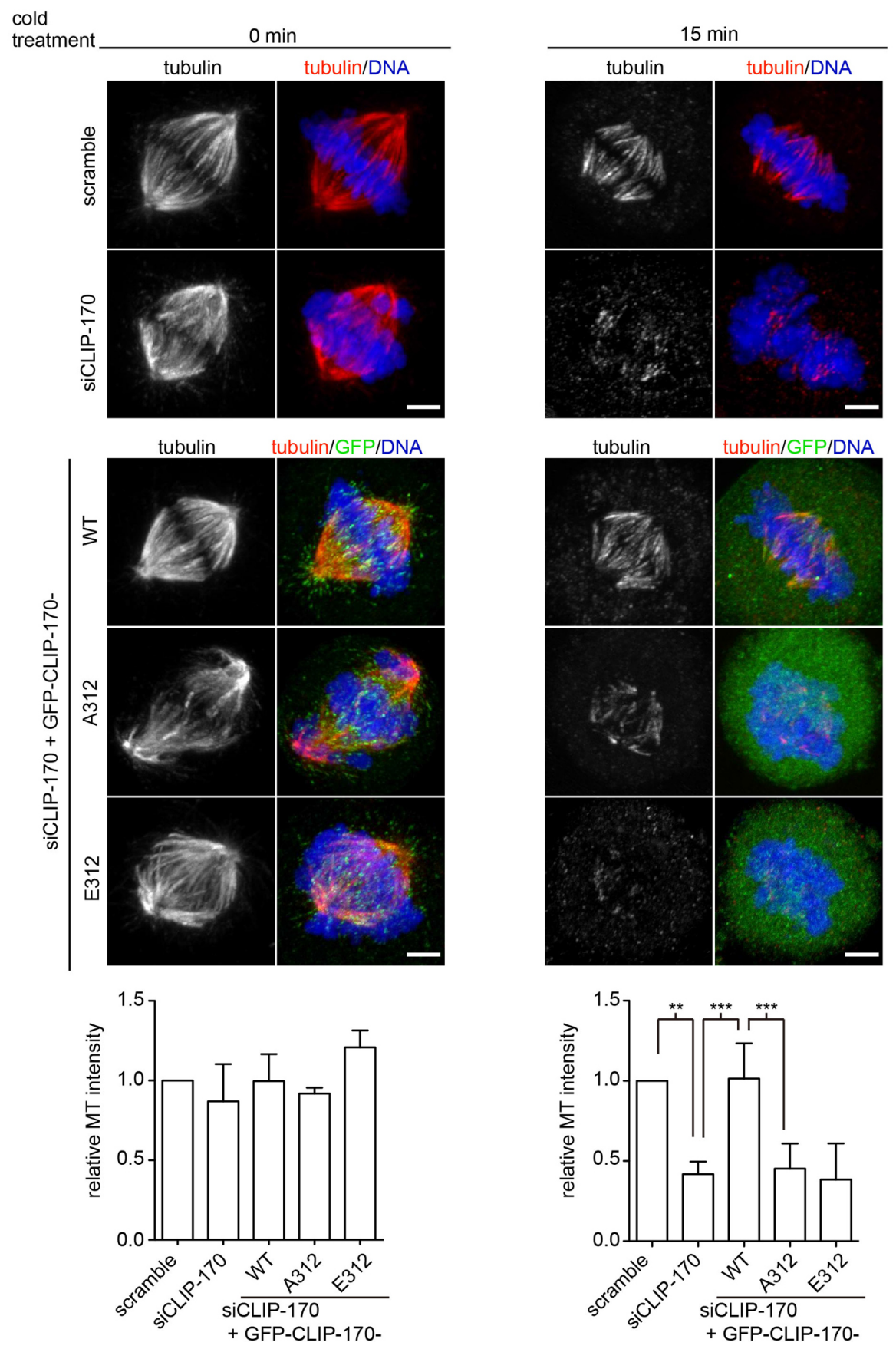

Fig. 7. CLIP-170 and its phosphorylation at Ser312 contribute to stable kinetochore/MT attachment. (A) Schematic representation of the experimental procedure (see Materials and Methods for details). HeLa cells were transfected with the indicated plasmids, cultured for $6 \mathrm{~h}$, and then transfected with control scramble or CLIP-170 siRNA. After $24 \mathrm{~h}$, the cells were treated with nocodazole for $16 \mathrm{~h}$ and released for 30 min followed by cold treatment ( 0 min or $15 \mathrm{~min})$ and immunostaining. (B) Cells were processed as described in A and stained with the indicated antibodies. CLIP-170 depletion significantly reduced the amount of cold-stable kMTs. The expression of CLIP-170 WT rescued the defect in the formation of cold-stable kMTs, but the expression of either nonphosphorylatable or phosphomimetic mutant failed to do so. The fluorescence intensity of the spindles was quantified and is shown as a ratio to the control cell value for each condition ( $0 \mathrm{~min}$ or $15 \mathrm{~min}$ ) in the lower graphs. Bars, $5 \mu \mathrm{m}$. The data represent the means $\pm \mathrm{SD}$ of more than three independent experiments. $\mathrm{n}>30$. $*{ }^{*} \mathrm{p}<0.01, * * * \mathrm{p}<0.001$ (one-way ANOVA, Tukey's HSD). The results are representative of at least four experiments. 
phosphorylates CLIP-170 at Ser312 in migrating fibroblasts (Nakano et al., 2010). Here, we demonstrate that during the cell cycle, the mitotic kinase Plk1 mediates CLIP-170 phosphorylation at the same serine residue. Although they phosphorylate the same site, the phosphorylation of Ser312 by these kinases has different results. In migrating cells, Ser312 is constitutively phosphorylated by AMPK, as an AMPK activator is unable to increase the phosphorylation level (Nakano et al., 2010). Meanwhile, we found that cell cycle progression transiently induces this phosphorylation (Fig. 1B). In migrating cells, the phosphomimetic CLIP170 Asp312 mutant fully rescued the defects caused by CLIP-170 depletion but failed to do so in mitotic cells (Fig. 6 and Fig. 7), suggesting that cell cycle progression, especially chromosome alignment, kinetochore/MT attachment, and/or stabilization, requires a strictly regulated phosphorylation and dephosphorylation cycle at Ser312 in CLIP-170 (see below). These observations raise the possibility that the difference in phosphorylation turnover ensures the divergent control of CLIP-170 activity, even if this turnover involves only a single phosphorylation site. Thus, the phosphorylation state of CLIP-170 is stringently controlled in a manner that is dependent on the cellular context.

Of note, $\mathrm{Li}$ et al. reported that Plk1 phosphorylates CLIP-170 at Ser195; this modification facilitates the complex formation between CLIP-170 and casein kinase 2, which leads to further phosphorylation in the C-terminal region of CLIP-170 (Li et al., 2010). Although we failed to reproduce Ser195 phosphorylation by Plk1 in vitro (Fig. 2D), we confirmed that the Plk1-mediated phosphorylation of CLIP-170 is required for proper kinetochore/MT attachment.

\section{Regulatory mechanisms that control CLIP-170 activity via phosphorylation}

The end-tracking of CLIP-170 requires the association of its N-terminal CAP-Gly domains with the acidic tails of both EBs and tubulin (Bieling et al., 2008; Dixit et al., 2009). The C-terminal region of CLIP-170 autonomously and intramolecularly inhibits these interactions, thereby diminishing end-tracking (Lansbergen et al., 2004). Accumulating evidence has shown that the third serine-rich region, which is adjacent to the CAP-Gly domain, also contributes to the end-tracking of CLIP-170 (Gupta et al., 2009, 2010). Using the N-terminal fragment of CLIP-170, we demonstrated that phosphorylation at Ser312 in the third serine-rich region reduced binding to MTs without affecting binding to EB3 or to its own C-terminal region (Fig. 4 and Fig. S3A). Furthermore, in vitro reconstitution showed that phosphorylation by Plk1 impaired the end-tracking ability of CLIP-170-NT (Fig. 5). Because CLIP-170 shows dynamic turnover within the growing ends of MTs in living cells (Dragestein et al., 2008), phosphorylation at Ser312 is most likely to decrease loading onto and residual time at the MT ends by modulating the binding of CLIP-170 to tubulin tails, independent of EB3 and its intramolecular association.

CLIP-170 has been proposed to stabilize MTs due to its anti-catastrophe activity in yeast (Brunner and Nurse, 2000) and rescue-enhancing activity in mammals (Komarova et al., 2002). However, in in vitro experiments where CLIP170 tracks the MT ends, many researchers, including ourselves, have failed to demonstrate these CLIP-170 activities (Bieling et al., 2008; Dixit et al., 2009; and data not shown), indicating missing factor(s) (i.e., factors other than EBs and tubulin) in in vitro reconstitution experiments. CLIP-170-binding CLASP proteins track the growing ends of MTs, which is dependent on EBs (Fig. S3C; Akhmanova et al., 2001; Mimori-Kiyosue et al., 2005; Honnappa et al., 2009), and promote MT rescue by recruiting tubulin dimers through its multiple TOG-like domains (Al-Bassam et al., 2010), although the effect of the association between CLIP-170 and CLASPs is not clear. We found that Plk1mediated phosphorylation in CLIP-170 reduced its binding to CLASP2 (Fig. S3A). A phosphomimetic mutation at Ser312 of CLIP-170 decreased its association with CLASP2 in cells (Fig. S3B). Furthermore, CLIP-170-NT facilitated CLASP2 recruitment to MTs, whereas phosphorylated CLIP-170-NT did so to a lesser degree (Fig. S3D). Taken together, these results indicate that CLIP-170 may control the dynamics of MT ends by forming an interactive network between +TIPs, including EBs and CLASPs, and that +TIP phosphorylation, such as that of CLIP-170 at Ser312, can modify the network that determines MT dynamics.

\section{Roles of CLIP-170 phosphorylation in kinetochore/MT attachment during mitosis}

What is the role of CLIP-170 phosphorylation by Plk1 during mitosis? CLIP-170 accumulates at prometaphase kinetochores, where Plk1 localizes and is activated (Petronczki et al., 2008) to form kinetochore/MT attachments (Dujardin et al., 1998; Tanenbaum et al., 2006; Li et al., 2010). During prometaphase, initial kinetochore/MT interactions are unstable and undergo turnover to correct inappropriate attachments until sister kinetochores establish bi-polar attachments, followed by kMT formation and chromosome alignment (Tanaka, 2010; Funabiki and Wynne, 2013). We showed that CLIP-170 localized to prometaphase kinetochores irrespective of the phosphorylation state of Ser312 (Fig. 6) and that phosphorylation by Plk1 diminished CLIP-170 binding to MTs (Fig. 4 and Fig. 5). Furthermore, our results demonstrated that a precise phosphorylation/ dephosphorylation cycle of CLIP-170 at Ser312 was required for stable kinetochore/MT attachment and proper chromosome alignment (Fig. 6 and Fig. 7). In addition, once kMTs are stably attached to kinetochores in metaphase, CLIP-170 is no longer detectable at the kinetochores 
and Plk1 activity is low at the kinetochores (Tanenbaum et al., 2006; Liu et al., 2012). Consistent with the role of Plk1 during prometaphase for kinetochore/MT attachment (Liu et al., 2012), we propose that CLIP-170 phosphorylation by Plk1 allows effective turnover of the initial kinetochore/MT attachments by adjusting CLIP-170 binding to MTs at the kinetochores and facilitates the establishment of bipolar attachments, followed by proper chromosome alignment.

In conclusion, in this study, we showed the dynamic regulation of CLIP-170 by phosphorylation, which depends on the cellular context. We propose that a precise phosphorylation/dephosphorylation cycle of CLIP-170 enables proper chromosome alignment by regulating the interaction between CLIP-170 and MTs in mitotic cells.

Acknowledgments. We thank R. Uehara, W. Li, and G. Goshima (Nagoya University) and K. Kasahara (Aichi Cancer Center Research Institute) for helpful discussions and sharing materials, and M. Dogterom and L. Laan (AMOLF, Netherlands) for developing the in vitro reconstitution assays. We also thank all members of the Kaibuchi lab for discussions and support. We acknowledge the Division of Medical Research Engineering of the Nagoya University Graduate School of Medicine (I. Mizuguchi, Y. Ito, M. Tanaka, K. Taki, and Y. Fujita) for allowing us to use ImageQuant LAS 4010 (GE Healthcare), DU800 spectrophotometer (Beckman Coulter), Optima MAX-XP ultracentrifuge (Beckman Coulter), and A1Rsi microscope (Nikon). We thank the Radioisotope Center Medical Branch of the Nagoya University School of Medicine (Technical Staff, N. Hamada and Y. Nakamura) for allowing us to perform the experiments with the radioisotopes. This work was supported by JST CREST to K.K., KAKENHI (20227006 to K.K., 20790225 to T.W.), GCOE to K.K., and SCF to T.W. M.K. was supported by the JSPS fellowship.

\section{References}

Akhmanova, A., Hoogenraad, C.C., Drabek, K., Stepanova, T., Dortland, B., Verkerk, T., Vermeulen, W., Burgering, B.M., De Zeeuw, C.I., Grosveld, F., and Galjart, N. 2001. Clasps are CLIP-115 and -170 associating proteins involved in the regional regulation of microtubule dynamics in motile fibroblasts. Cell, 104: 923-935.

Akhmanova, A. and Steinmetz, M.O. 2008. Tracking the ends: a dynamic protein network controls the fate of microtubule tips. Nat. Rev. Mol. Cell Biol., 9: 309-322.

Al-Bassam, J., Kim, H., Brouhard, G., van Oijen, A., Harrison, S.C., and Chang, F. 2010. CLASP promotes microtubule rescue by recruiting tubulin dimers to the microtubule. Dev. Cell, 19: 245-258.

Bieling, P., Kandels-Lewis, S., Telley, I.A., van Dijk, J., Janke, C., and Surrey, T. 2008. CLIP-170 tracks growing microtubule ends by dynamically recognizing composite EB1/tubulin-binding sites. J. Cell Biol., 183: $1223-1233$.

Brunner, D. and Nurse, P. 2000. CLIP170-like tip1p spatially organizes microtubular dynamics in fission yeast. Cell, 102: 695-704.

Coquelle, F.M., Caspi, M., Cordelieres, F.P., Dompierre, J.P., Dujardin, D.L., Koifman, C., Martin, P., Hoogenraad, C.C., Akhmanova, A., Galjart, N., De Mey, J.R., and Reiner, O. 2002. LIS1, CLIP-170's key to the dynein/dynactin pathway. Mol. Cell. Biol., 22: 3089-3102.

DeLuca, J.G. 2010. Kinetochore-microtubule dynamics and attachment stability. Methods Cell Biol., 97: 53-79.

Dixit, R., Barnett, B., Lazarus, J.E., Tokito, M., Goldman, Y.E., and Holzbaur, E.L. 2009. Microtubule plus-end tracking by CLIP-170 requires EB1. Proc. Natl. Acad. Sci. USA, 106: 492-497.
Dragestein, K.A., van Cappellen, W.A., van Haren, J., Tsibidis, G.D., Akhmanova, A., Knoch, T.A., Grosveld, F., and Galjart, N. 2008. Dynamic behavior of GFP-CLIP-170 reveals fast protein turnover on microtubule plus ends. J. Cell Biol., 180: 729-737.

Dujardin, D., Wacker, U.I., Moreau, A., Schroer, T.A., Rickard, J.E., and De Mey, J.R. 1998. Evidence for a role of CLIP-170 in the establishment of metaphase chromosome alignment. J. Cell Biol., 141: 849-862.

Folker, E.S., Baker, B.M., and Goodson, H.V. 2005. Interactions between CLIP-170, tubulin, and microtubules: implications for the mechanism of Clip-170 plus-end tracking behavior. Mol. Biol. Cell, 16: 5373-5384.

Fukata, M., Watanabe, T., Noritake, J., Nakagawa, M., Yamaga, M., Kuroda, S., Matsuura, Y., Iwamatsu, A., Perez, F., and Kaibuchi, K. 2002. Rac1 and Cdc42 capture microtubules through IQGAP1 and CLIP-170. Cell, 109: 873-885.

Funabiki, H. and Wynne, D.J. 2013. Making an effective switch at the kinetochore by phosphorylation and dephosphorylation. Chromosoma, 122: $135-158$.

Galjart, N. 2010. Plus-end-tracking proteins and their interactions at microtubule ends. Curr. Biol., 20: R528-537.

Gell, C., Bormuth, V., Brouhard, G.J., Cohen, D.N., Diez, S., Friel, C.T., Helenius, J., Nitzsche, B., Petzold, H., Ribbe, J., Schaffer, E., Stear, J.H., Trushko, A., Varga, V., Widlund, P.O., Zanic, M., and Howard, J. 2010. Microtubule dynamics reconstituted in vitro and imaged by single-molecule fluorescence microscopy. Methods Cell Biol., 95: 221245.

Goto, H., Yasui, Y., Nigg, E.A., and Inagaki, M. 2002. Aurora-B phosphorylates Histone $\mathrm{H} 3$ at serine 28 with regard to the mitotic chromosome condensation. Genes Cells, 7: 11-17.

Gouveia, S.M. and Akhmanova, A. 2010. Cell and molecular biology of microtubule plus end tracking proteins: end binding proteins and their partners. Int. Rev. Cell Mol. Biol., 285: 1-74.

Gupta, K.K., Paulson, B.A., Folker, E.S., Charlebois, B., Hunt, A.J., and Goodson, H.V. 2009. Minimal plus-end tracking unit of the cytoplasmic linker protein CLIP-170. J. Biol. Chem., 284: 6735-6742.

Gupta, K.K., Joyce, M.V., Slabbekoorn, A.R., Zhu, Z.C., Paulson, B.A., Boggess, B., and Goodson, H.V. 2010. Probing interactions between CLIP-170, EB1, and microtubules. J. Mol. Biol., 395: 1049-1062.

Honnappa, S., Okhrimenko, O., Jaussi, R., Jawhari, H., Jelesarov, I., Winkler, F.K., and Steinmetz, M.O. 2006. Key interaction modes of dynamic +TIP networks. Mol. Cell, 23: 663-671.

Honnappa, S., Gouveia, S.M., Weisbrich, A., Damberger, F.F., Bhavesh, N.S., Jawhari, H., Grigoriev, I., van Rijssel, F.J., Buey, R.M., Lawera, A., Jelesarov, I., Winkler, F.K., Wuthrich, K., Akhmanova, A., and Steinmetz, M.O. 2009. An EB1-binding motif acts as a microtubule tip localization signal. Cell, 138: 366-376.

Howard, J. and Hyman, A.A. 2003. Dynamics and mechanics of the microtubule plus end. Nature, 422: 753-758.

Inoko, A., Matsuyama, M., Goto, H., Ohmuro-Matsuyama, Y., Hayashi, Y., Enomoto, M., Ibi, M., Urano, T., Yonemura, S., Kiyono, T., Izawa, I., and Inagaki, M. 2012. Trichoplein and Aurora A block aberrant primary cilia assembly in proliferating cells. J. Cell Biol., 197: 391-405.

Komarova, Y.A., Akhmanova, A.S., Kojima, S., Galjart, N., and Borisy, G.G. 2002. Cytoplasmic linker proteins promote microtubule rescue in vivo. J. Cell Biol., 159: 589-599.

Lansbergen, G., Komarova, Y., Modesti, M., Wyman, C., Hoogenraad, C.C., Goodson, H.V., Lemaitre, R.P., Drechsel, D.N., van Munster, E., Gadella, T.W., Jr., Grosveld, F., Galjart, N., Borisy, G.G., and Akhmanova, A. 2004. Conformational changes in CLIP-170 regulate its binding to microtubules and dynactin localization. J. Cell Biol., 166: 1003-1014.

Lee, H.S., Komarova, Y.A., Nadezhdina, E.S., Anjum, R., Peloquin, J.G., Schober, J.M., Danciu, O., van Haren, J., Galjart, N., Gygi, S.P., Akhmanova, A., and Borisy, G.G. 2010. Phosphorylation controls auto- 
inhibition of cytoplasmic linker protein-170. Mol. Biol. Cell, 21: 26612673.

Li, H., Liu, X.S., Yang, X., Wang, Y., Turner, J.R., and Liu, X. 2010. Phosphorylation of CLIP-170 by Plk1 and CK2 promotes timely formation of kinetochore-microtubule attachments. EMBO J., 29: 2953-2965.

Li, W., Miki, T., Watanabe, T., Kakeno, M., Sugiyama, I., Kaibuchi, K., and Goshima, G. 2011. EB1 promotes microtubule dynamics by recruiting Sentin in Drosophila cells. J. Cell Biol., 193: 973-983.

Liu, D., Davydenko, O., and Lampson, M.A. 2012. Polo-like kinase-1 regulates kinetochore-microtubule dynamics and spindle checkpoint silencing. J. Cell Biol., 198: 491-499.

Liu, X. and Erikson, R.L. 2002. Activation of Cdc2/cyclin B and inhibition of centrosome amplification in cells depleted of Plk1 by siRNA. Proc. Natl. Acad. Sci. USA, 99: 8672-8676.

Lomakin, A.J., Semenova, I., Zaliapin, I., Kraikivski, P., Nadezhdina, E., Slepchenko, B.M., Akhmanova, A., and Rodionov, V. 2009. CLIP-170dependent capture of membrane organelles by microtubules initiates minus-end directed transport. Dev. Cell, 17: 323-333.

Macurek, L., Lindqvist, A., Lim, D., Lampson, M.A., Klompmaker, R., Freire, R., Clouin, C., Taylor, S.S., Yaffe, M.B., and Medema, R.H. 2008. Polo-like kinase-1 is activated by aurora A to promote checkpoint recovery. Nature, 455: 119-123.

Maurer, S.P., Bieling, P., Cope, J., Hoenger, A., and Surrey, T. 2011. GTPgammaS microtubules mimic the growing microtubule end structure recognized by end-binding proteins (EBs). Proc. Natl. Acad. Sci. USA, 108: 3988-3993.

Maurer, S.P., Fourniol, F.J., Bohner, G., Moores, C.A., and Surrey, T. 2012. EBs recognize a nucleotide-dependent structural cap at growing microtubule ends. Cell, 149: 371-382.

Mimori-Kiyosue, Y., Grigoriev, I., Lansbergen, G., Sasaki, H., Matsui, C., Severin, F., Galjart, N., Grosveld, F., Vorobjev, I., Tsukita, S., and Akhmanova, A. 2005. CLASP1 and CLASP2 bind to EB1 and regulate microtubule plus-end dynamics at the cell cortex. J. Cell Biol., 168: $141-153$.

Mishima, M., Maesaki, R., Kasa, M., Watanabe, T., Fukata, M., Kaibuchi, K., and Hakoshima, T. 2007. Structural basis for tubulin recognition by cytoplasmic linker protein 170 and its autoinhibition. Proc. Natl. Acad. Sci. USA, 104: 10346-10351.

Nakano, A., Kato, H., Watanabe, T., Min, K.D., Yamazaki, S., Asano, Y., Seguchi, O., Higo, S., Shintani, Y., Asanuma, H., Asakura, M., Minamino, T., Kaibuchi, K., Mochizuki, N., Kitakaze, M., and Takashima, S. 2010. AMPK controls the speed of microtubule polymerization and directional cell migration through CLIP-170 phosphorylation. Nat. Cell Biol., 12: 583-590.

Petronczki, M., Lénárt, P., and Peters, J.M. 2008. Polo on the Rise-from Mitotic Entry to Cytokinesis with Plk1. Dev. Cell, 14: 646-659.

Pierre, P., Scheel, J., Rickard, J.E., and Kreis, T.E. 1992. CLIP-170 links endocytic vesicles to microtubules. Cell, 70: 887-900.
Rickard, J.E. and Kreis, T.E. 1990. Identification of a novel nucleotidesensitive microtubule-binding protein in HeLa cells. J. Cell Biol., 110: $1623-1633$.

Rickard, J.E. and Kreis, T.E. 1991. Binding of pp170 to microtubules is regulated by phosphorylation. J. Biol. Chem., 266: 17597-17605.

Rickard, J.E. and Kreis, T.E. 1996. CLIPs for organelle-microtubule interactions. Trends Cell Biol., 6: 178-183.

Seki, A., Coppinger, J.A., Jang, C.Y., Yates, J.R., and Fang, G. 2008. Bora and the kinase Aurora a cooperatively activate the kinase Plk1 and control mitotic entry. Science, 320: 1655-1658.

Shiromizu, T., Goto, H., Tomono, Y., Bartek, J., Totsukawa, G., Inoko, A., Nakanishi, M., Matsumura, F., and Inagaki, M. 2006. Regulation of mitotic function of Chk1 through phosphorylation at novel sites by cyclin-dependent kinase 1 (Cdk1). Genes Cells, 11: 477-485.

Slep, K.C. 2010. Structural and mechanistic insights into microtubule end-binding proteins. Curr. Opin. Cell Biol., 22: 88-95.

Tai, C.Y., Dujardin, D.L., Faulkner, N.E., and Vallee, R.B. 2002. Role of dynein, dynactin, and CLIP-170 interactions in LIS1 kinetochore function. J. Cell Biol., 156: 959-968.

Tanaka, T.U. 2010. Kinetochore-microtubule interactions: steps towards bi-orientation. EMBO J., 29: 4070-4082.

Tanenbaum, M.E., Galjart, N., van Vugt, M.A., and Medema, R.H. 2006. CLIP-170 facilitates the formation of kinetochore-microtubule attachments. EMBO J., 25: 45-57.

Telley, I.A., Bieling, P., and Surrey, T. 2011. Reconstitution and quantification of dynamic microtubule end tracking in vitro using TIRF microscopy. Methods Mol. Biol., 777: 127-145.

Watanabe, T., Noritake, J., and Kaibuchi, K. 2005. Regulation of microtubules in cell migration. Trends Cell Biol., 15: 76-83.

Watanabe, T., Noritake, J., Kakeno, M., Matsui, T., Harada, T., Wang, S., Itoh, N., Sato, K., Matsuzawa, K., Iwamatsu, A., Galjart, N., and Kaibuchi, K. 2009. Phosphorylation of CLASP2 by GSK-3beta regulates its interaction with IQGAP1, EB1 and microtubules. J. Cell Sci., 122: 2969-2979.

Wong, J. and Fang, G. 2006. HURP controls spindle dynamics to promote proper interkinetochore tension and efficient kinetochore capture. $J$. Cell Biol., 173: 879-891.

Yamaguchi, T., Goto, H., Yokoyama, T., Sillje, H., Hanisch, A., Uldschmid, A., Takai, Y., Oguri, T., Nigg, E.A., and Inagaki, M. 2005. Phosphorylation by Cdk1 induces Plk1-mediated vimentin phosphorylation during mitosis. J. Cell Biol., 171: 431-436.

Yang, X., Li, H., Liu, X.S., Deng, A., and Liu, X. 2009. Cdc2-mediated phosphorylation of CLIP-170 is essential for its inhibition of centrosome reduplication. J. Biol. Chem., 284: 28775-28782.

(Received for publication, January 6, 2014, accepted, January 16, 2014 and published online, January 22, 2014) 